\title{
Evaluation of a Nonpeptidic Ligand for Imaging of Cholecystokinin 2 Receptor-Expressing Cancers
}

\author{
Charity Wayua and Philip S. Low \\ Department of Chemistry, Purdue University, West Lafayette, Indiana
}

Tumor-specific targeting ligands were recently exploited to deliver both imaging and therapeutic agents selectively to cancer tissues in vivo. Because the cholecystokinin 2 receptor (CCK2R) is overexpressed in various human cancers (e.g., lung, medullary thyroid, pancreatic, colon, and gastrointestinal stromal tumors) but displays limited expression in normal tissues, natural ligands of CCK2R were recently explored for use in the imaging of CCK2R-expressing cancers. Unfortunately, the results from these studies revealed not only that the peptidic CCK2R ligands were unstable in vivo but also that the ligands that mediated good uptake by tumor tissues also promoted a high level of retention of the radioimaging agent in the kidneys, probably because of capture of the conjugates by peptidescavenging receptors. In an effort to reduce the normal organ retention of CCK2R-targeted drugs, we synthesized a nonpeptidic ligand of CCK2R and examined its specificity for CCK2R both in vitro and in vivo. Methods: Nonpeptidic agonists and antagonists of CCK2R described in the literature were evaluated for their affinities and specificities for CCK2R. Z-360, a benzodiazepine-derived CCK2R antagonist with subnanomolar affinity, was selected for complexation to ${ }^{99 \mathrm{~m} T c}$ via multiple spacers. After synthesis and purification, 4 complexes with different physicochemical properties were evaluated for binding to CCK2R-transfected HEK 293 cells. The best conjugate, termed CRL-3-99mTc, was injected into mice bearing CCK2R tumor xenografts and examined by y scintigraphy and SPECT/CT. The uptake of the conjugate in various organs was also quantified by tissue resection and y counting. Results: CRL3-99mTc was shown to bind with low nanomolar affinity to CCK2R in vitro and was localized to tumor tissues in athymic nu/nu mice implanted with CCK2R-expressing tumors. At $4 \mathrm{~h}$ after injection, tumor uptake was measured at $12.0 \pm 2.0$ percentage injected dose per gram of tissue. Conclusion: Because the uptake of CRL-3-99mTc by nonmalignant tissues was negligible and retention in the kidneys was only transient, we suggest that CRL-3- ${ }^{99 m T c}$ may be a useful radioimaging agent for the detection, sizing, and monitoring of CCK2Rexpressing tumors.

Key Words: tumor-targeted therapy; radioimaging of tumors; cholecystokinin 2/gastrin receptor; cancer imaging; nonpeptide; antagonist

\section{J Nucl Med 2015; 56:113-119}

DOI: 10.2967/jnumed.114.144998

\footnotetext{
Received Jun. 30, 2014; revision accepted Nov. 7, 2014.

For correspondence or reprints contact: Philip S. Low, Department of Chemistry, Purdue University, 720 Clinic Dr., West Lafayette, IN 47907.

E-mail: plow@purdue.edu

Published online Dec. 11, 2014

COPYRIGHT $\odot 2015$ by the Society of Nuclear Medicine and Molecular Imaging, Inc.
}

$\mathbf{T}$ he overexpression of specific receptors on pathologic cell surfaces has been exploited for the functional imaging of disease activity in both humans (1-4) and animal models of human disease (5-7). One such receptor, the cholecystokinin (CCK) 2 receptor (gastrin receptor; CCK2R), is a G-protein-coupled receptor that is normally expressed in the central nervous system and the cells of the gastric mucosa (8). In the central nervous system, CCK regulates appetite, pain, anxiety, and wakefulness $(9,10)$. In the gastrointestinal tract, the closely related gastrin peptides modulate the growth and differentiation of the gastric mucosa and the secretion of acid, bile, and digestive enzymes (11).

CCK2R is also overexpressed in several cancers, in which it has been reported to mediate the stimulation of cancer cell growth and migration (12,13), metastasis (14), and survival (15). Cancers shown to upregulate CCK2R include malignancies of the thyroid (16); lung (17); pancreas, ovary, and brain (18); stomach and gastrointestinal stroma (19); and colon (20). Recently, a novel splice variant of CCK2R that is tumor-specific and often coexpressed with the normal CCK2R splice form in malignant cells $(20,21)$ was also identified. This splice variant, designated CCK2i4svR, results from the retention of intron 4 , which yields a modified receptor containing a 64-amino-acid insertion in the third cytoplasmic domain. Importantly, this modified CCK2R is constitutively active (22), leading to increased basal cell proliferation and enhanced tumorigenicity, even in the absence of a ligand (23).

The limited expression of CCK2R in normal tissues, coupled with the overexpression of CCK2R and CCK $2 \mathrm{i} 4 \mathrm{svR}$ in various cancers, has rendered the receptor an attractive candidate for targeted imaging and therapy of malignant disease. Indeed, radioactive conjugates of CCK and gastrin have already been explored for use in the imaging of CCK2R-overexpressing tumors in mice (24-26). Unfortunately, the results from these studies revealed inadequacies that suggested the need for improved CCK2R targeting ligands. First, gastrin peptides that exhibit high levels of tumor uptake are also compromised by high levels of retention in the kidneys, whereas CCK peptides that display low levels of kidney uptake also show little retention in tumors (27-29). As a consequence, the development of CCK2R-targeted peptides for radiotherapeutic applications has been hampered by concerns that the targeted radiation may simultaneously cause damage to the kidneys. Second, the fact that gastrin and CCK, like most other peptide hormones, are rapidly degraded by peptidases in the serum and on cell surfaces (30) has raised concern that attached cytotoxic cargos may be released during circulation, resulting in their nonspecific distribution into CCK2R-negative tissues. Third, CCKand gastrin-related peptides are prone to oxidation at a methionine residue, leading to the loss of receptor binding affinity and their consequent dissemination into receptor-negative tissues (30). 
Finally, CCK- and gastrin-related peptides can stimulate the growth, proliferation, and survival of cancer cells, rendering the tumor-targeted imaging agent an unwanted promoter of tumor growth.

To avoid the unwanted consequences associated with the use of a peptidic agonist for tumor imaging, we designed a nonpeptidic CCK2R targeting ligand and used it to deliver a ${ }^{99 m} \mathrm{Tc}$-based radioimaging agent to $\mathrm{CCK} 2 \mathrm{R}$-expressing cancers in tumor-bearing mice. We report here that this CCK2R targeting ligand promoted the tumor-specific accumulation of an attached radioimaging agent with a low level of accumulation in normal tissues, suggesting a possible use for the targeted imaging agent in the detection, localization, and staging of CCK2R-expressing tumors.

\section{MATERIALS AND METHODS}

\section{Chemicals}

All amino acids and resins were purchased from Chem Impex Intl. Sodium pertechnetate $\left({ }^{99 \mathrm{~m}} \mathrm{Tc}\right.$ ) was obtained from Cardinal Health. HC Matrigel was purchased from BD Biosciences. Stannous chloride, sodium glucoheptonate, diisopropylethylamine (DIPEA), piperidine, dimethylformamide (DMF), isopropyl alcohol, and all other reagents were purchased from Sigma-Aldrich.

\section{Cell Culture and Animal Husbandry}

Two HEK 293 cell lines, one transfected with CCK2R (HEK 293 CCK2R cells) and the other expressing CCK2i4svR (HEK 293 CCK2i4svR cells), were kind gifts from Dr. Mark Hellmich (University of Texas Medical Branch). Cells were cultured in Dulbecco modified Eagle medium (GIBCO) supplemented with $10 \%$ fetal bovine serum, G418 disulfate (Sigma-Aldrich; $400 \mu \mathrm{g} / \mathrm{mL}$ ), and $1 \%$ penicillin-streptomycin at $37^{\circ} \mathrm{C}$ in a humidified $95 \%$ air $-5 \% \mathrm{CO}_{2}$ atmosphere. Athymic female $n u / n u$ mice were purchased from Harlan Laboratories, housed in a sterile environment on a standard 12-h lightdark cycle, and maintained on normal rodent chow. All animal pro-

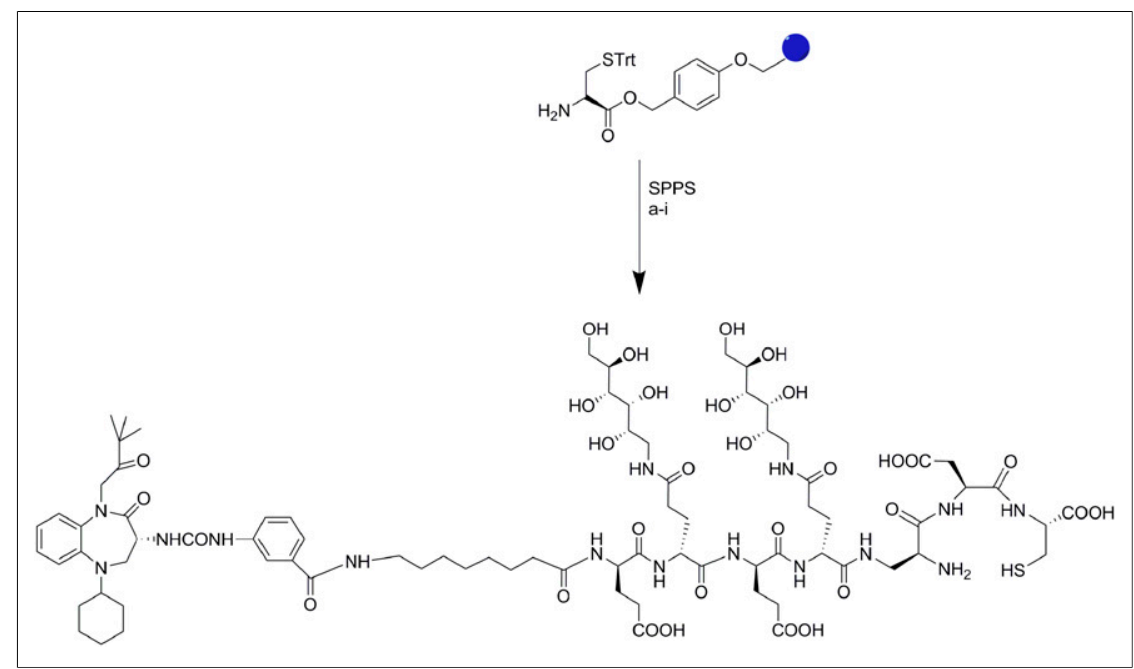

FIGURE 1. Solid-phase synthesis of CRL-3. All reactions were conducted under $\mathrm{N}_{2}$ or $\mathrm{Ar}$ at room temperature. Reagents and conditions in reactions a-i were as follows: (a) Fmoc-Asp(OtBu)-OH, HATU, DIPEA, 4 h; (b) (i) 20\% piperidine-DMF, 10 min; (ii) Fmoc-diaminopropionic (DAP) acid, HATU, DIPEA, 4 h; (c) (i) 20\% piperidine-DMF, 10 min; (ii) 3,4,5,6-di-isopropylidene-1-amino-deoxy(FmocGlu-OH)-D-glucitol, HATU, DIPEA, 4 h; (d) (i) 20\% piperidine-DMF, 10 min; (ii) Fmoc-Glu(OtBu)-OH, HATU, DIPEA, 4 h; (e) (i) 20\% piperidine-DMF, $10 \mathrm{~min}$; (ii) 3,4,5,6-di-isopropylidene-1-amino-deoxy (Fmoc-Glu-OH)-D-glucitol, HATU, DIPEA, 4 h; (f) (i) 20\% piperidine-DMF, 10 min; (ii) Fmoc-Glu(OtBu)OH, HATU, DIPEA, 4 h; (g) (i) 20\% piperidine-DMF, 10 min; (ii) Fmoc-8-amino-octanoic acid, HATU, DIPEA, 4 h; (h) (i) 20\% piperidine-DMF, 10 min; (ii) Z-360, HATU, DIPEA, overnight; (i) cleavage in trifluoroacetic acid- $\mathrm{H}_{2} \mathrm{O}-$ TIPS-EDT (92.5:2.5:2.5:2.5), $30 \mathrm{~min}$. EDT = ethanedithiol; SPPS = solidphase peptide synthesis; STrt $=$ trityl protected cysteine; TIPS $=$ triisopropylsilane. cedures were approved by the Purdue Animal Care and Use Committee in accordance with National Institutes of Health guidelines.

\section{Synthesis of Radioimaging Agents}

Z-360, a CCK2R antagonist, was synthesized in accordance with previously reported methods $(31,32)$ and linked via the desired spacer ence $\beta$-L-diaminopropionic acid, L-aspartic acid (L-Asp), and the desired spacer was then added (Fig. 1).

Next, the Z-360 ligand was linked to the terminal amine, and the final conjugate was cleaved from the resin and purified as described later. In brief, acid-sensitive Wang resin (Sigma-Aldrich) loaded with reacted with Fmoc-Asp(OtBu)-OH $(0.265 \mathrm{mmol}), 2-(1 \mathrm{H}-7$-azabenzotriazol-1-yl)-1,1,3,3-tetramethyl uranium hexafluorophosphate (HATU; (0.265 mmol), and diisopropylethylamine $(1.06 \mathrm{mmol})$ to yield ${ }^{9 \mathrm{~m}} \mathrm{Tc}$ chelating moiety. The chelator was then conjugated to tions were performed under an argon atmosphere. Fmoc-protecting groups were removed after each coupling step under standard conditions (20\% piperidine in DMF). Release of the partially deprotected conjugate from the polymeric support was finally accomplished by treatment with a solution of $92.5 \%$ trifluoroacetic acid, $2.5 \%$ 1,2-ethanedithiol, $2.5 \%$ triisopropylsilane, and $2.5 \%$ deionized water. This reaction also resulted in the simultaneous removal of all $t$-butyl-, $t$-butoxycarbonyl-, and trityl-protecting groups.

The crude product was purified by preparative reverse-phase high-performance liquid chromatography with a gradient mobile phase of $20 \mathrm{mM}$ ammonium acetate buffer and acetonitrile and a solvent gradient of 5\% acetonitrile to $80 \%$ acetonitrile over $30 \mathrm{~min}$ (xTerra $\mathrm{C} 18$; Waters; $10 \mu \mathrm{m} ; 19 \times 250 \mathrm{~mm}$ ). Elution of the conjugate was monitored at a wavelength of $280 \mathrm{~nm}$, and the identities of the eluted compounds were analyzed by liquid chromatography-mass spectrometry and matrixassisted laser desorption ionization. Formulation and radiolabeling of the conjugates with ${ }^{99 \mathrm{~m}} \mathrm{Tc}$ were performed in accordance with previously described methods (33).

\section{Binding Affinity and Specificity of CCK2R Ligand- ${ }^{99 m}$ Tc-Labeled Conjugates}

HEK 293 CCK2R and HEK 293 CCK2i4svR cells were seeded onto 24-well plates and grown to $80 \%-90 \%$ confluence for $48-72 \mathrm{~h}$. Spent medium in each well was replaced with $0.5 \mathrm{~mL}$ of fresh medium containing $0.5 \%$ bovine serum albumin, and increasing concentrations of the radioconjugate were added. After incubation for $1 \mathrm{~h}$ at $37^{\circ} \mathrm{C}$, the cells were rinsed with incubation solution $(2 \times 1.0 \mathrm{~mL})$ and resuspended in $0.5 \mathrm{~mL}$ of $0.25 \mathrm{~N} \mathrm{NaOH}$ before radioactivity was counted with a $\gamma$ counter. The dissociation 


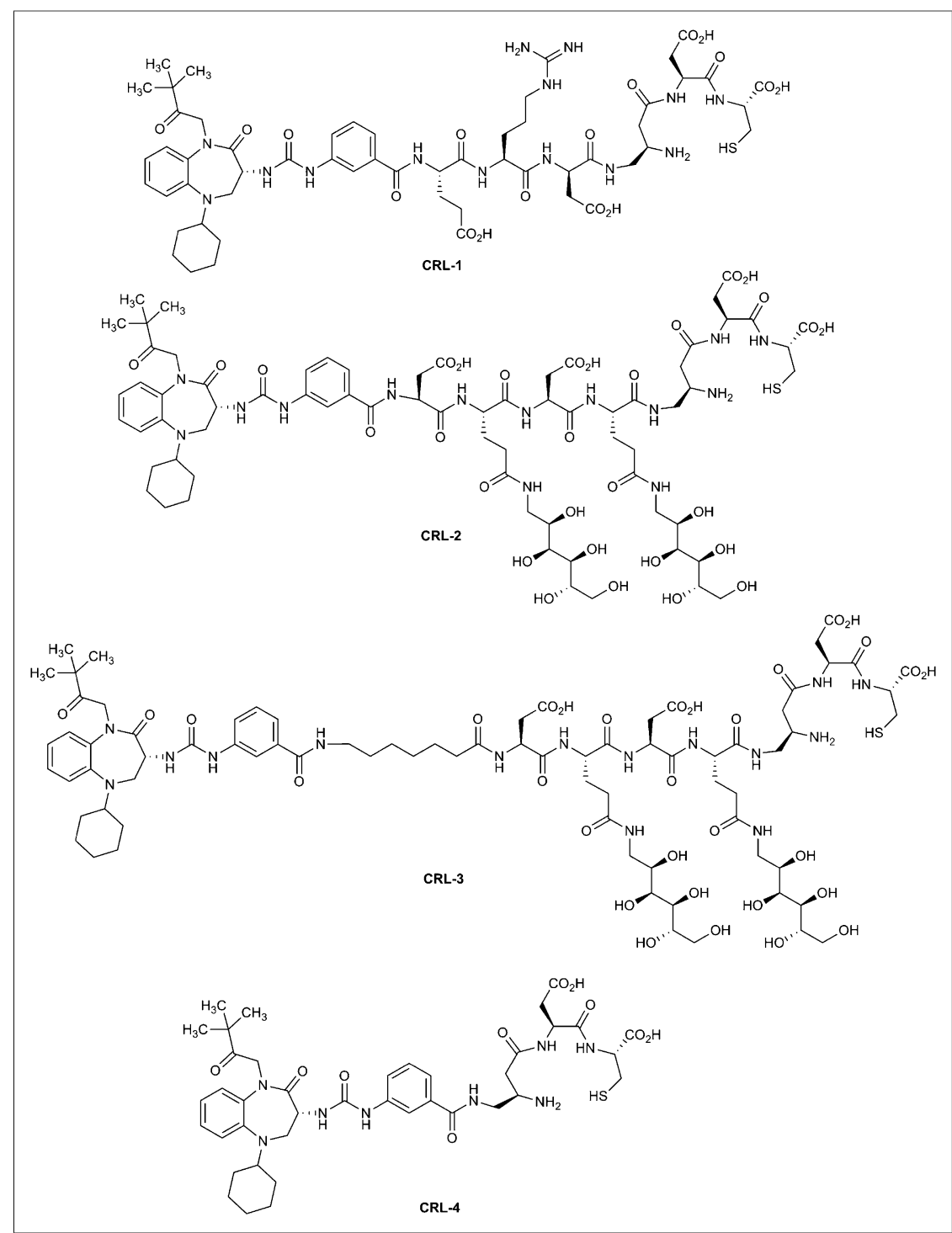

FIGURE 2. Structures of CCK receptor ligand-targeted radioimaging conjugates.

\section{Analysis of Tumor-Bearing Mice}

Six-week-old female $n u / n u$ mice were inoculated subcutaneously in their shoulders with either HEK 293 CCK2R or HEK 293 CCK2i4svR cells (in 50\% HC Matrigel-Dulbecco modified Eagle medium; $5.0 \times 10^{6}$ cells per mouse) with a 25 -gauge needle. The growth of the tumors was measured in 2 perpendicular directions every $2 \mathrm{~d}$ with a caliper, and the volumes of the tumors were calculated with the formula $0.5 \times L \times W^{2}$, where $L$ is the measurement of the longest axis and $W$ is the measurement of the axis perpendicular to $L$, in millimeters. Radiotracer biodistribution studies were performed 12-18 d after tumor cell implantation, when the tumors had reached volumes of approximately $400-500 \mathrm{~mm}^{3}$. The mice were randomly assigned to different treatment groups and injected intravenously with the desired ${ }^{99 \mathrm{~m} T c-l a b e l e d ~ c o n j u g a t e ~}(10 \mathrm{nmol}$; $5.55 \mathrm{MBq}[150 \mu \mathrm{Ci}]$ in $100 \mu \mathrm{L}$ of phosphatebuffered saline). At various time points $(0.5$, $2,4,8$, and $24 \mathrm{~h}$ after injection), the animals were sacrificed by $\mathrm{CO}_{2}$ asphyxiation, and imaging was performed with a KODAK Image Station. The parameters used for radioimaging were as follows: acquisition time, $2 \mathrm{~min}$; f-stop, 4; focal plane, 7; field of view, 200; and binning, 4. For white-light imaging, the parameters were as follows: acquisition time, $0.05 \mathrm{~s}$; f-stop, 11; focal plane, 7; field of view, 200; and no binning. SPECT/CT imaging was performed with micro-SPECT II/CT (MILabs).

\section{Biodistribution of Radioimaging Agents}

After radioimaging, each animal was dissected, and selected organs and tissues were collected into preweighed tubes and subjected to $\gamma$ counting. Counts per minute were decay corrected and converted to percentage injected dose per gram of wet tissue. constant $\left(\mathrm{K}_{\mathrm{d}}\right)$ was calculated by plotting cell-bound radioactivity as a function of the concentration of the radiotracer in the medium, with the assumption of a noncooperative single-site-binding equilibrium.

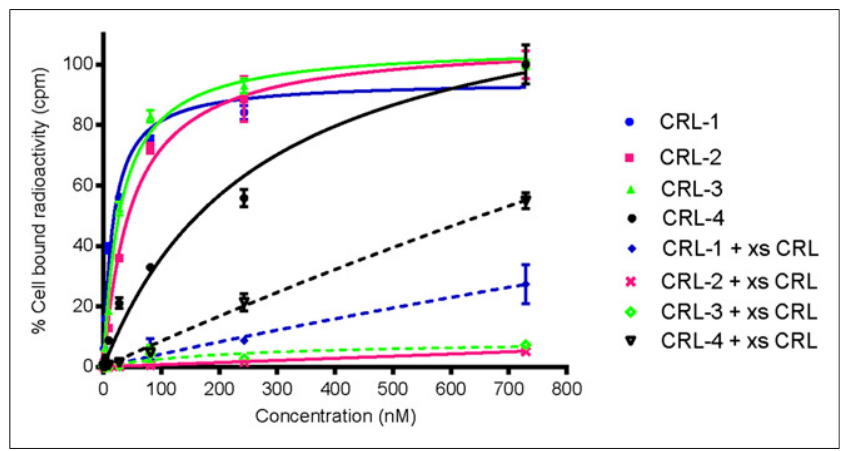

FIGURE 3. In vitro binding isotherms of CRL-1, CRL-2, and CRL-3 for HEK 293 CCK2R cells in absence (solid lines) and presence (broken lines) of 100 -fold molar excess of unlabeled CCK receptor ligand (xs CRL). Error bars indicate SDs $(n=3)$.

\section{RESULTS}

Because of the limited expression of CCK2R in normal tissues and the overexpression of CCK2R in various tumors, we elected to develop a CCK2R-targeted ${ }^{99 m}$ Tc-chelate complex that would not be encumbered by the specificity and stability problems associated with previous CCK2R-targeted peptidic radioimaging agents. After an extensive literature search, we found a small molecule antagonist, Z-360 (35,36), that binds CCK2R with subnanomolar affinity and high selectivity relative to the cholecystokinin 1 receptor (CCK1R) $\left(\mathrm{K}_{\mathrm{d}}, 0.47 \mathrm{nmol} / \mathrm{L}\right.$; selectivity relative to CCK1R, 672). Preferential binding to CCK2R was important because CCK1R is expressed at high levels in several normal tissues (37). Moreover, ${ }^{99 \mathrm{~m}} \mathrm{Tc}$ was chosen as the radiolabel because of its widespread availability, low cost, and short half-life $(6.02 \mathrm{~h})$.

For construction of the optimal CCK2R-targeted radioimaging agent, Z-360 was attached to a previously described ${ }^{99 \mathrm{~m}} \mathrm{Tc}$ chelating agent (33) via various spacers designed to both improve water solubility and prevent chelate complex interference with ligand binding. The final conjugates selected for comparison are shown in Figure 2. CCK receptor ligand 1 (CRL-1) contains a simple 
TABLE 1

Binding Affinities of CCK Receptor Ligand-Targeted Radioimaging Agents in HEK 293 Cells

\begin{tabular}{lcc}
\hline Compound & $\begin{array}{c}\mathrm{K}_{\mathrm{d}}(\mathrm{nM}) \text { of HEK } \\
293 \text { CCK2R cells }\end{array}$ & $\begin{array}{c}\mathrm{K}_{\mathrm{d}}(\mathrm{nM}) \text { of HEK 293 } \\
\text { CCK2i4svR cells }\end{array}$ \\
\hline CRL-1 & 16 & \\
CRL-2 & 46 & 31 \\
CRL-3 & 30 & 4 \\
CRL-4 & 270 &
\end{tabular}

${ }^{*} \mathrm{~K}_{\mathrm{d}}$ could not be determined for compounds CRL-1 and CRL-4 in HEK 293 CCK2i4svR cells because of very high levels of nonspecific binding of these compounds and significantly smaller numbers of receptors on these cells.

tripeptide spacer. Because scavenger receptors in the kidneys and liver can bind diverse peptidic conjugates $(38,39)$, the peptide spacer in CRL-1 was replaced with a less readily scavenged peptidosaccharide spacer in CCK receptor ligand 2 (CRL-2) (39). Next, to ensure sufficient separation between the CCK receptor ligand and its tethered radiochelate, CCK receptor ligand 3 (CRL-3) was designed with the same spacer as CRL-2, but an octanoyl moiety was inserted before the peptidosaccharide spacer. Finally, to assist with evaluation of the impact of spacer length, CCK receptor ligand 4 (CRL-4) was prepared with no spacer.

Because the affinity of a ligand for its receptor can often be compromised by attachment to its cargo, the binding affinity of each of the conjugates for both CCK2R and CCK2i4svR was examined in vitro. For this purpose, HEK 293 CCK2R and HEK 293 CCK2i4svR cells were incubated with increasing concentrations of each radioactive conjugate, both in the presence and in the absence of a 100-fold excess of unlabeled CCK receptor ligand. Next, after washing was done to remove unbound conjugate, cellassociated radioactivity was determined by $\gamma$ counting. As shown in Figure 3 and Table 1, CRL-1, CRL-2, and CRL-3 all showed high affinities for both cell lines, with binding constants in the

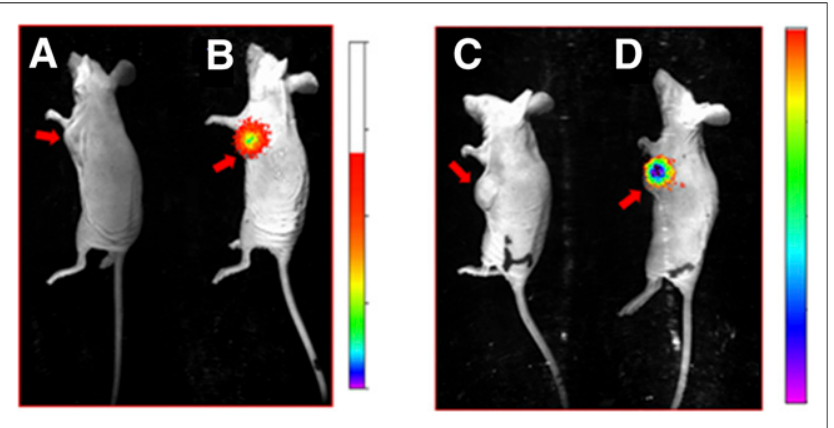

FIGURE 4. Evaluation of specificity of CRL-3-99mTc for CCK2Rexpressing tumors in mice. Overlay of whole-body radioimages (rainbow colors) on white-light photographs of nu/nu mice bearing HEK 293 CCK2R (A and B) and HEK 293 CCK2i4svR (C and D) tumor xenografts in presence $(A$ and $C)$ and absence $(B$ and $D)$ of 100 -fold molar excess of CRL-3. At $2 \mathrm{~h}$ after tail vein administration of $5.55 \mathrm{MBq}$ of CRL3-99mTc, mice were euthanized, and kidneys were shielded with lead plate to allow easier visualization of radioconjugate in other tissues. Red arrows indicate locations of tumors. nanomolar range. In contrast, CRL-4, which contained no spacer, displayed low affinities for both cell lines; this result confirmed the impact of spacer length on ligand affinity.

To determine the fraction of conjugate uptake that was receptormediated, we performed parallel experiments in the presence of a 100-fold molar excess of unlabeled CCK receptor ligand to competitively block receptor binding. As shown in Figure 3, CRL2 and CRL-3 showed quantitative competition on the addition of excess ligand, whereas CRL-4 and CRL-1 showed approximately $50 \%$ and approximately $20 \%$ residual nonspecific binding, respectively. We suspect that the strong hydrophobicity of Z-360 favored membrane association for all conjugates and that only the enhanced steric bulk and hydrophilicity of the peptidosaccharide spacers were sufficient to prevent nonspecific membrane association. The slightly higher affinity of CRL-3 than of CRL-2 may have been due to the increased separation of the binding ligand from the bulky peptidosaccharide spacer; however, additional subsite interactions may also have contributed to this difference in affinity. On

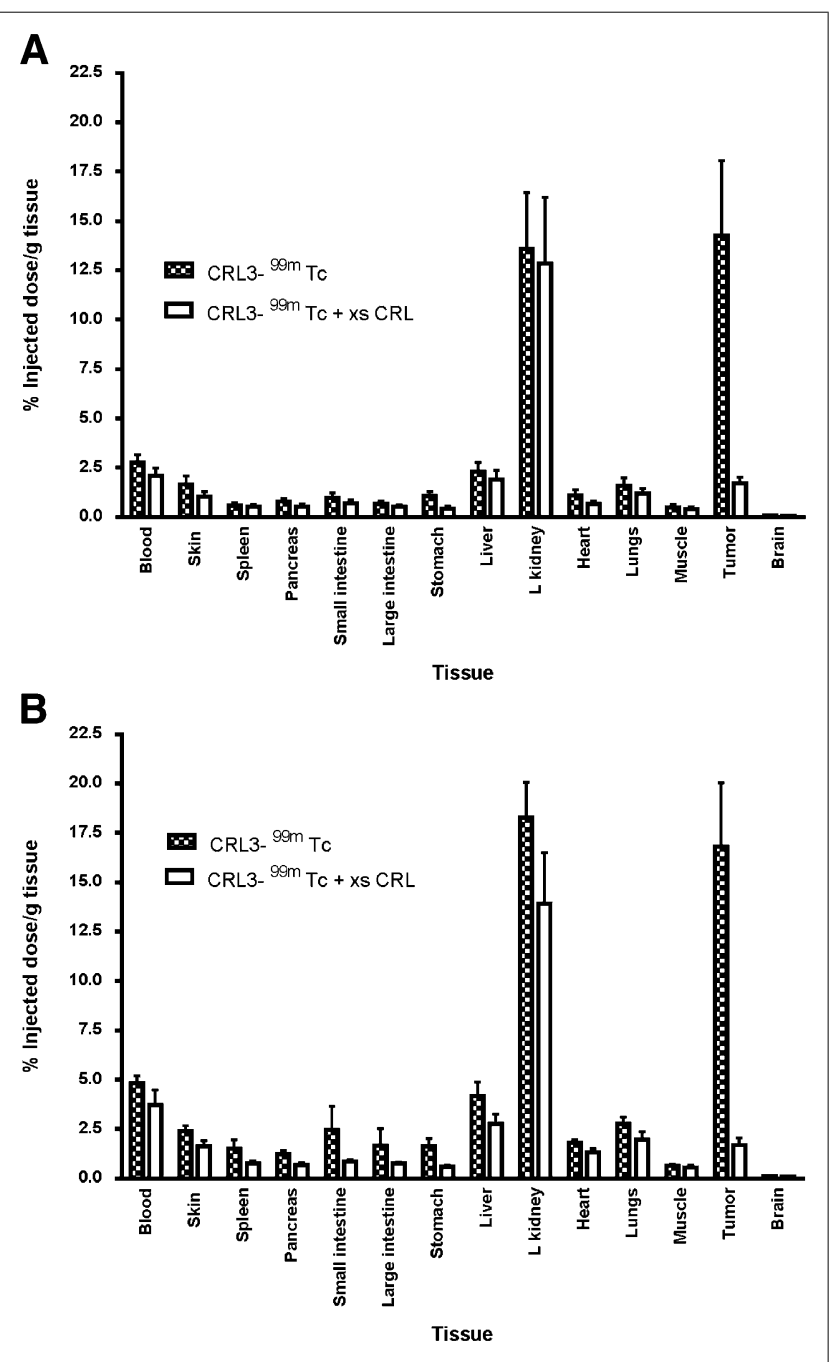

FIGURE 5. Tissue distribution of CRL-3- ${ }^{99 \mathrm{mT}} \mathrm{Tc}$ in nu/nu mice bearing HEK 293 CCK2R (A) and HEK 293 CCK2i4svR (B) tumor xenografts $2 \mathrm{~h}$ after injection of $5.55 \mathrm{MBq}$ of CRL-3-99mTc. Error bars represent SDs ( $n=5$ mice per group). L kidney = left kidney; $x \mathrm{CRL}=$ excess of unlabeled CCK receptor ligand. 


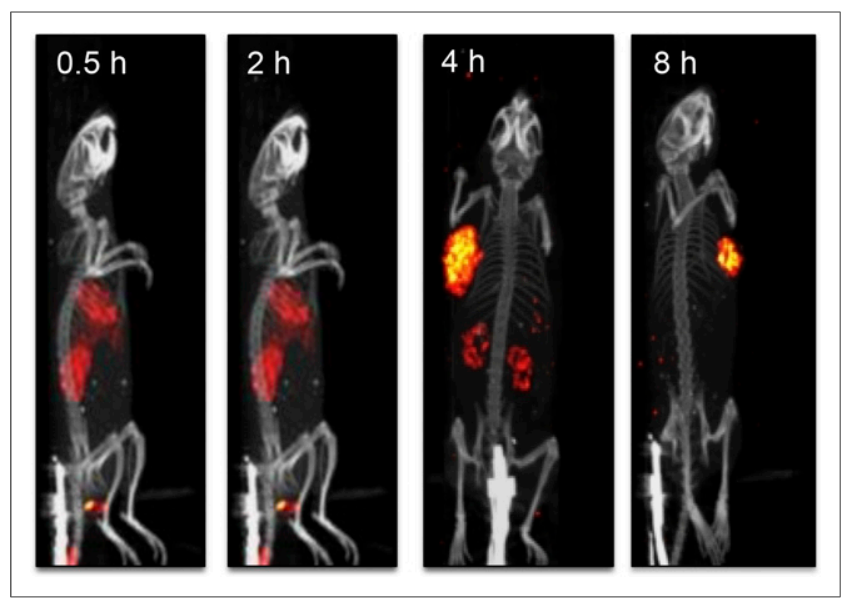

FIGURE 6. Examination of rate of clearance of CRL-3-99mTc from tumor-bearing mice with SPECT/CT. Mice implanted with HEK 293 CCK2R tumor xenografts were injected in tail vein with $5.55 \mathrm{MBq}$ of CRL-3-99mTc and imaged 0.5, 2, 4, and $8 \mathrm{~h}$ after injection.

the basis of these data, CRL-3 was selected for further testing in mice bearing both CCK2R and CCK2i4svR tumor xenografts.

The in vivo targeting and specificity of CRL-3-99m Tc were evaluated by injecting CRL-3-99mTc intravenously into athymic $n u / n u$ mice bearing HEK 293 CCK2R and HEK 293 CCK2i4svR tumor xenografts. As shown in Figure 4, CRL-3- ${ }^{99 m}$ Tc was observed to accumulate in HEK 293 CCK2R and HEK 293 CCK2i4svR tumors, with little or no accumulation in other tissues except the kidneys (14.25 percentage injected dose per gram of tissue $2 \mathrm{~h}$ after injection). Preinjection of the mice with a 100 -fold excess of unlabeled CCK receptor ligand was found to block tumor uptake, indicating that the accumulation of the radiotracer in the malignant mass was receptor-mediated (Fig. 5). In contrast, the uptake of CRL-3- ${ }^{99 m}$ Tc in the kidneys was not affected by excess CRL-3 (Fig. 5), suggesting that the presence of CRL3- ${ }^{99 \mathrm{~m}} \mathrm{Tc}$ in the kidneys did not depend on CCK2R expression.

Because small molecules are often excreted via the liver or kidneys (40), we examined whether the uptake of CRL-3-99m $\mathrm{Tc}$ in the liver and kidneys may have been transient. As shown in the SPECT/CT images in Figure 6, the CRL-3-99m Tc content in the liver and kidneys decreased over time, whereas uptake in the tumor mass was relatively stable.

By $24 \mathrm{~h}$ after tail vein injection (Table 2), the tumor-to-normal tissue ratios of CRL-3-99m Tc in the muscle, heart, skin, blood, liver, and spleen were 90, 83, 30, 61, 4, and 14, respectively. These data suggest that CCK2R-expressing tissues retained accumulated CRL-3- ${ }^{99 m} \mathrm{Tc}$ in substantially greater quantities and, therefore, that any radiation damage to normal tissues should be minimal.

\section{DISCUSSION}

The overexpression of CCK2R in many malignant cells and the limited expression of CCK2R in normal tissues have suggested that CCK2R-targeted radioemitters may be attractive candidates for the radioimaging of many human cancers. However, the very high uptake of natural ligands of CCK2R by the kidneys (in some cases, $\sim 60 \%$ of the injected dose) (29) has strongly discouraged further investigation of peptides for this application. The objective of this study therefore was to explore the development of nonpeptidic ligands for the imaging of CCK2R-expressing tumors. We showed here that a small organic antagonist of CCK2R could be exploited to yield high-resolution images of CCK2R-expressing tumors, especially when time was allowed for the clearance of CCK receptor ligand conjugates from the liver and kidneys. In addition to the avoidance of hepatic and renal

TABLE 2

Tissue Distribution of CRL-3-99mTc After Subcutaneous Injection of Mice with HEK 293 Cells Expressing CCK2R

\begin{tabular}{|c|c|c|c|c|c|}
\hline \multirow[b]{2}{*}{ Organ } & \multicolumn{5}{|c|}{ Tissue distribution at: } \\
\hline & $0.5 \mathrm{~h}$ & $2 \mathrm{~h}$ & $4 \mathrm{~h}$ & $8 \mathrm{~h}$ & $24 \mathrm{~h}$ \\
\hline Blood & $5.8 \pm 2.7$ & $1.9 \pm 0.97$ & $1.7 \pm 0.39$ & $0.24 \pm 0.19$ & $0.10 \pm 0.06$ \\
\hline Skin & $2.3 \pm 1.2$ & $1.0 \pm 0.41$ & $1.1 \pm 0.23$ & $0.42 \pm 0.22$ & $0.21 \pm 0.13$ \\
\hline Spleen & $2.1 \pm 1.2$ & $0.69 \pm 0.70$ & $1.4 \pm 0.41$ & $0.65 \pm 0.42$ & $0.45 \pm 0.39$ \\
\hline Pancreas & $1.3 \pm 0.62$ & $0.69 \pm 0.84$ & $0.29 \pm 0.19$ & $0.10 \pm 0.06$ & $0.053 \pm 0.03$ \\
\hline Small intestine & $1.2 \pm 0.63$ & $0.52 \pm 0.28$ & $0.52 \pm 0.08$ & $0.19 \pm 0.10$ & $0.13 \pm 0.08$ \\
\hline Large intestine & $1.0 \pm 0.52$ & $0.46 \pm 0.20$ & $0.53 \pm 0.11$ & $0.24 \pm 0.16$ & $0.13 \pm 0.08$ \\
\hline Stomach & $1.2 \pm 0.61$ & $0.58 \pm 0.32$ & $0.56 \pm 0.16$ & $0.24 \pm 0.15$ & $0.15 \pm 0.09$ \\
\hline Liver & $6.7 \pm 3.6$ & $2.5 \pm 2.4$ & $4.1 \pm 0.64$ & $1.9 \pm 1.2$ & $1.4 \pm 1.4$ \\
\hline Left kidney & $11.2 \pm 6.1$ & $8.4 \pm 4.6$ & $13.4 \pm 2.1$ & $6.0 \pm 3.1$ & $2.6 \pm 1.6$ \\
\hline Right kidney & $11.4 \pm 6.3$ & $7.6 \pm 4.4$ & $12.8 \pm 1.8$ & $5.7 \pm 3.0$ & $2.6 \pm 1.7$ \\
\hline Heart & $2.3 \pm 1.5$ & $0.68 \pm 0.47$ & $0.59 \pm 0.18$ & $0.14 \pm 0.078$ & $0.076 \pm 0.05$ \\
\hline Lungs & $3.2 \pm 1.7$ & $1.2 \pm 0.55$ & $1.0 \pm 0.23$ & $0.29 \pm 0.15$ & $0.18 \pm 0.12$ \\
\hline Muscle & $0.8 \pm 0.4$ & $0.29 \pm 0.096$ & $0.3 \pm 0.04$ & $0.093 \pm 0.048$ & $0.070 \pm 0.08$ \\
\hline Brain & $0.13 \pm 0.07$ & $0.051 \pm 0.02$ & $0.053 \pm 0.016$ & $0.064 \pm 0.12$ & $0.0076 \pm 0.005$ \\
\hline Tumor & $7.4 \pm 4.6$ & $8.1 \pm 5.1$ & $12.0 \pm 2.0$ & $8.5 \pm 4.9$ & $6.3 \pm 3.7$ \\
\hline
\end{tabular}

Uptake of radioactivity is expressed as percentage injected dose per gram of wet tissue $(n=5$ at $0.5,4,8$, and $24 \mathrm{~h} ; n=10$ at $2 \mathrm{~h})$. 
retention with nonpeptidic CCK2R ligands, these ligands may enable improved serum stability (35).

To evaluate the effect of spacer chemistry on CCK2R targeting, we synthesized and evaluated 4 different conjugates with distinct spacers. Although each conjugate recognized both CCK2R and CCK2i4svR, CRL-3 displayed the highest affinity for both forms of the receptor, whereas CRL-4 showed the lowest affinity for the 2 types of receptor. Moreover, CRL- 4 and, to a lesser extent, CRL-1 were both compromised by a high level of nonspecific binding, suggesting the need for a hydrophilic spacer to offset the hydrophobicity of the core ligand, Z-360. As shown in Figure 3, the binding of both peptidosaccharide-containing spacers could be nearly quantitatively blocked on the addition of excess ligand, suggesting almost exclusive receptor-mediated binding by those conjugates. CRL-3-99m Tc also showed favorable pharmacokinetics in vivo, with rapid blood and kidney clearance and sustained tumor retention.

If CCK receptor ligand-targeted radiotherapeutic and chemotherapeutic agents display similar biodistribution profiles, then the possible use of CCK receptor ligands for targeting therapeutic payloads should not be ignored. The observed tissue distribution of CRL-3- ${ }^{99 m} \mathrm{Tc}$ implied that toxicity may be localized to the tumor, with negligible damage to normal tissues. Moreover, because kidney and liver accumulation was only transient, the selection of a radiotherapeutic agent with a longer half-life may allow clearance of the radioactivity from the kidneys before significant decay of the radiotherapeutic conjugate in the tumor has occurred. In both radiotherapeutic and chemotherapeutic applications, prior imaging of patients with CRL-3-99m Tc may also prove useful for preselecting patients whose tumors express sufficient CCK2R to benefit from CCK receptor ligand-targeted therapy.

\section{CONCLUSION}

In addition to the targeting of radioimaging and chemotherapeutic agents, CCK receptor ligands may also be exploited for the delivery of other imaging modalities (e.g., PET, CT, and optical and MR imaging contrast agents) selectively to CCK2R-expressing tumors. Furthermore, as shown for other tumor-targeted ligands $(4,7)$, CCK receptor ligands may also be developed for the delivery of fluorescent probes for use in fluorescence-guided resection of malignant masses during surgery (32). Fluorescent CCK receptor ligands may similarly be adapted for use in the detection and capture of circulating tumor cells in the blood of cancer patients. In brief, if the tumor selectivity of CRL-3-99m Tc is maintained with other conjugates of CCK receptor ligands, then various future clinical applications can be envisioned.

\section{DISCLOSURE}

The costs of publication of this article were defrayed in part by the payment of page charges. Therefore, and solely to indicate this fact, this article is hereby marked "advertisement" in accordance with 18 USC section 1734 . No potential conflict of interest relevant to this article was reported.

\section{ACKNOWLEDGMENTS}

We thank Dr. Venkatesh Chelvam for help with the KODAK imaging system, Aaron Taylor at the Purdue University Bioimaging Center for assistance with SPECT/CT, and Dr. Mini Thomas for helpful discussions.

\section{REFERENCES}

1. Ivancević V, Wormann B, Nauck C, et al. Somatostatin receptor scintigraphy in the staging of lymphomas. Leuk Lymphoma. 1997;26:107-114.

2. Nejmeddine F, Raphael M, Martin A, Le Roux G, Moretti JL, CaillatVigneron $\mathrm{N} .{ }^{67} \mathrm{Ga}$ scintigraphy in B-cell non-Hodgkin's lymphoma: correlation of ${ }^{67} \mathrm{Ga}$ uptake with histology and transferrin receptor expression. J Nucl Med. 1999;40:40-45.

3. Behr TM, Behe MP. Cholecystokinin-B/gastrin receptor-targeting peptides for staging and therapy of medullary thyroid cancer and other cholecystokinin-B receptor-expressing malignancies. Semin Nucl Med. 2002;32:97-109.

4. van Dam GM, Themelis G, Crane LMA, et al. Intraoperative tumor-specific fluorescence imaging in ovarian cancer by folate receptor- $\alpha$ targeting: first inhuman results. Nat Med. 2011;17:1315-1319.

5. Zhang J, Razavian M, Tavakoli S, et al. Molecular imaging of vascular endothelial growth factor receptors in graft arteriosclerosis. Arterioscler Thromb Vasc Biol. 2012;32:1849-1855.

6. Vaitilingam B, Chelvam V, Kularatne SA, Poh S, Ayala-Lopez W, Low PS. A folate receptor- $\alpha$-specific ligand that targets cancer tissue and not sites of inflammation. J Nucl Med. 2012;53:1127-1134.

7. Kularatne SA, Zhou Z, Yang J, et al. Design, synthesis, and preclinical evaluation

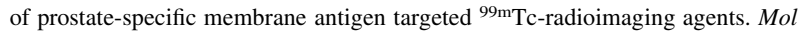
Pharm. 2009;6:790-800.

8. Mantyh CRP, Pappas TN, Vigna SR. Localization of cholecystokinin A and cholecystokinin $\mathrm{B} /$ gastrin receptors in the canine upper gastrointestinal tract. Gastroenterology. 1994;107:1019-1030.

9. Little TJ, Horowitz M, Feinle-Bisset C. Role of cholecystokinin in appetite control and body weight regulation. Obes Rev. 2005;6:297-306.

10. Bowers ME, Choi DC, Ressler KJ. Neuropeptide regulation of fear and anxiety: implications of cholecystokinin, endogenous opioids, and neuropeptide Y. Physiol Behav. 2012;107:699-710.

11. Dufresne M, Seva C, Fourmy D. Cholecystokinin and gastrin receptors. Physiol Rev. 2006;86:805-847.

12. Noble PJ, Wilde G, White MR. Stimulation of gastrin-CCKB receptor promotes migration of gastric AGS cells via multiple paracrine pathways. Am J Physiol Gastrointest Liver Physiol. 2003;284:G75-G84.

13. Oikonomou E, Buchfelder M, Adams EF. Cholecystokinin (CCK) and CCK receptor expression by human gliomas: evidence for an autocrine/paracrine stimulatory loop. Neuropeptides. 2008;42:255-265.

14. Wroblewski LE, Pritchard DM, Carter S, et al. Gastrin-stimulated gastric epithelial cell invasion: the role and mechanism of increased matrix metalloproteinase 9 expression. Biochem J. 2002;365:873-879.

15. Watson SA, Grabowska AM, El-Zaatari M, et al. Gastrin: active participant or bystander in gastric carcinogenesis? Nat Rev Cancer. 2006;6:936-946.

16. Reubi JC, Waser B. Unexpected high incidence of cholecystokinin B/gastrin receptors in human medullary thyroid carcinomas. Int J Cancer. 1996;67: 644-647.

17. Sethi T, Herget M, Wu SV, et al. CCK-A and CCK-B receptors are expressed in small cell lung cancer lines and mediate $\mathrm{Ca}^{2+}$ mobilization and clonal growth. Cancer Res. 1993;53:5208-5213.

18. Reubi JCSJ, Waser B. Cholecystokinin (CCK)-A and CCK-B/gastrin receptors in human tumors. Cancer Res. 1997;57:1377-1386.

19. Hur K, Lee HJ, Park DJ, et al. Expression of gastrin and its receptor in human gastric cancer tissues. J Cancer Res Clin Oncol. 2006;132:85-91.

20. Körner M, Reubi JC, Miller LJ. CCK(2) receptor splice variant with intron 4 retention in human gastrointestinal and lung tumours. J Cell Mol Med. 2010; 14:933-943.

21. Smith JP, Verderame MF, McLaughlin P, et al. Characterization of the CCKC (cancer) receptor in human pancreatic cancer. Int J Mol Med. 2002;10: 689-694.

22. Olszewska-Pazdrak B, Townsend CM Jr, Hellmich MR. Agonist-independent activation of Src tyrosine kinase by a cholecystokinin-2 (CCK2) receptor splice variant. J Biol Chem. 2004;279:40400-40404.

23. Chao C, Goluszko E, Lee Y-T, et al. Constitutively active CCK2 receptor splice variant increases Src-dependent HIF-1a expression and tumor growth. Oncogene. 2007;26:1013-1019.

24. Laverman P, Joosten L, Eek A, et al. Comparative biodistribution of $12{ }^{111} \mathrm{In}$ labelled gastrin/CCK2 receptor-targeting peptides. Eur J Nucl Med Mol Imaging. 2011;38:1410-1416.

25. Laverman $P$, Roosenburg $S$, Gotthardt $M$, et al. Targeting of a CCK(2) receptor splice variant with ${ }^{111}$ In-labelled cholecystokinin-8 (CCK8) and ${ }^{111} \mathrm{In}$-labelled minigastrin. Eur J Nucl Med Mol Imaging. 2008;35:386-392.

26. Aloj L, Aurilio M, Rinaldi V, et al. Comparison of the binding and internalization properties of 12 DOTA-coupled and ${ }^{111}$ In-labelled CCK2/gastrin receptor 
binding peptides: a collaborative project under COST Action BM0607. Eur J Nucl Med Mol Imaging. 2011;38:1417-1425.

27. Behr TM, Jenner N, Radetzky S, et al. Targeting of cholecystokinin-B/gastrin receptors in vivo: preclinical and initial clinical evaluation of the diagnostic and therapeutic potential of radiolabelled gastrin. Eur J Nucl Med. 1998;25:424-430.

28. Behr TM, Béhé M, Angerstein C, et al. Cholecystokinin-B/gastrin receptor binding peptides: preclinical development and evaluation of their diagnostic and therapeutic potential. Clin Cancer Res. 1999;5(10 suppl):3124s-3138s.

29. Béhé M, Kluge G, Becker W, et al. Use of polyglutamic acids to reduce uptake of radiometal-labeled minigastrin in the kidneys. J Nucl Med. 2005;46:1012-1015.

30. Ocak M, Helbok A, Rangger C, et al. Comparison of biological stability and metabolism of CCK2 receptor targeting peptides: a collaborative project under COST BM0607. Eur J Nucl Med Mol Imaging. 2011;38:1426-1435.

31. Lauffer DJ, Mullican MD. A practical synthesis of (S) 3-tert-butoxycarbonylamino2-oxo-2,3,4,5-tetrahydro-1,5-benzodiazepine-1-acetic acid methyl ester as a conformationally restricted dipeptido-mimetic for caspase-1 (ICE) inhibitors. Bioorg Med Chem Lett. 2002;12:1225-1227.

32. Wayua C, Low PS. Evaluation of a cholecystokinin 2 receptor-targeted near-infrared dye for fluorescence-guided surgery of cancer. Mol Pharm. 2014;11:468-476.

33. Leamon CP, Parker MA, Vlahov IR, et al. Synthesis and biological evaluation of EC20: a new folate-derived, ${ }^{99 \mathrm{~m}} \mathrm{Tc}$-based radiopharmaceutical. Bioconjug Chem. 2002;13:1200-1210.
34. Vlahov IR, You F, Wang Y, et al. Carbohydrate-based synthetic approach to control toxicity profiles of folate-drug conjugates. J Org Chem. 2010;75: 3685-3691.

35. Kawasaki D, Emori Y, Eta R, et al. Effect of Z-360, a novel orally active CCK-2/ gastrin receptor antagonist on tumor growth in human pancreatic adenocarcinoma cell lines in vivo and mode of action determinations in vitro. Cancer Chemother Pharmacol. 2008;61:883-892.

36. Grabowska AM, Morris TM, McKenzie AJ, et al. Pre-clinical evaluation of a new orally-active CCK-2R antagonist, Z-360, in gastrointestinal cancer models. Regul Pept. 2008;146:46-57.

37. Monstein HJ, Nylander AG, Salehi A, et al. Cholecystokinin-A and cholecystokinin$\mathrm{B} /$ gastrin receptor mRNA expression in the gastrointestinal tract and pancreas of the rat and man: a polymerase chain reaction study. Scand J Gastroenterol. 1996;31: 383-390.

38. Vegt E, Melis M, Eek A, et al. Renal uptake of different radiolabelled peptides is mediated by megalin: SPECT and biodistribution studies in megalin-deficient mice. Eur J Nucl Med Mol Imaging. 2011;38:623-632.

39. Leelahavanichkul A, Bocharov AV, Kurlander R, et al. Class B scavenger receptor types I and II and CD36 targeting improves sepsis survival and acute outcomes in mice. J Immunol. 2012;188:2749-2758.

40. Maack T, Johnson V, Kan T, et al. Renal filtration, transport, and metabolism of low molecular weight proteins: a review. Kidney Int. 1979;16:251-270. 